\title{
HAMARTOMA E HIPERPLASIA ESTROMAL PSEUDOANGIOMATOSA DE LA MAMA, DIAGNÓSTICOS DIFERENCIALES INFRECUENTES DE CÁNCER DE MAMA*
}

\author{
Drs. Gladys Ibáñez R. ${ }^{1}$, Manuel Figueroa G. ${ }^{2}$, Omar Chávez M. ${ }^{2}$, \\ Fernando Olguín H. ${ }^{2}$, Virginia Leiva C. ${ }^{3}$, Marisol Guerrero G. ${ }^{3}$ \\ 1 Unidad de Patología Mamaria Complejo Hospitalario San José. \\ 2 Departamento de Cirugía Hospital Clínico de la Universidad de Chile. \\ 3 Servicio de Anatomía Patológica Complejo Hospitalario San José. \\ Santiago, Chile.
}

\begin{abstract}
Hamartoma and nodular pseudoangiomatous stromal hyperplasia. Differential diagnosis

Introduction: Hamartoma and nodular pseudoangiomatous stromal hyperplasia (PASH) are inusual breast tumor entities, however, can simulate breast cancer. The only accurate tool is preoperative biopsy. Surgical treatment is curative with low recurrence rate. Objective: To present a case of mammary hamartoma associated with PASH whose initial presentation was suggestive of probable malignancy. Case Report: Woman, 44 years old, admitted to controls because of probable malignant breast disease, mammography was reported as BIRADS 0, breast ultrasound as BIRADS U.S: 5, CORE biopsy reported PASH. Partial mastectomy was performed. On delayed biopsy mammary hamartoma with extensive stromal hyperplasia pseudoangiomatosa was diagnosed. Discussion: PASH and hamartomas are diseases with no specific clinical presentation, radiological findings may be pathognomonic of hamartoma, while not in PASH. In the reported case the pathological study showed an association of these conditions, described in a $16 \%$ to $71 \%$ breast hamartoma cases. The analysis of the entire surgical specimen is essential for a definitive diagnosis.
\end{abstract}

Key words: Hamartoma, nodular pseudoangiomatous stromal hyperplasia.

\section{Resumen}

Introducción: Los hamartomas y la hiperplasia estromal pseudoangiomatosa nodular (PASH) son entidades infrecuentes en la patología tumoral mamaria, sin embargo, pueden simular al cáncer de mama. La única herramienta certera preoperatoria es la biopsia. El tratamiento quirúrgico es curativo con bajo índice de recurrencia. Objetivo: El objetivo de este trabajo es exponer un caso de hamartoma mamario con PASH

*Recibido el 13 de mayo de 2013 y aceptado para publicación el 2 de agosto de 2013.

Los autores no refieren conflictos de interés.

Correspondencia: Dr. Manuel Figueroa G.

Av. Santos Dumont 999, Santiago, Chile.

manuelfigueroa.gi@gmail.com 
asociado cuya presentación inicial fue sugerente de probable patología maligna. Caso Clínico: Mujer de 44 años, ingresa a controles por probable patología mamaria maligna, la mamografía fue informada como BIRADS 0, la ecografía mamaria como BIRADS US: 5, la biopsia CORE informó PASH. Se realizó mastectomía parcial objetivando en la biopsia diferida un hamartoma mamario con extensa hiperplasia estromal pseudoangiomatosa. Discusión: Los hamartomas y la PASH son patologías sin presentación clínica específica, los hallazgos radiológicos del hamartoma pueden ser patognomónicos, no así en la PASH; en el caso expuesto el estudio anatomopatológico demostró una asociación de estas patologías, la cual se describe desde un 16\% a un $71 \%$ de los casos de hamartoma mamario. El análisis de toda la pieza quirúrgica es fundamental para establecer un diagnóstico definitivo.

Palabras clave: Hamartoma mamario, hiperplasia estromal seudoangiomatosa.

\section{Introducción}

Los hamartomas y la hiperplasia estromal pseudoangiomatosa nodular son entidades infrecuentes en la patología tumoral mamaria. El hamartoma mamario es una entidad benigna y con criterios diagnósticos histológicos poco específicos. La Hiperplasia Estromal Pseudoangiomatosa de la mama (PASH) es una entidad clínica con dos formas de presentación, como incidentaloma o como patología principal (PASH nodular), siendo esta última bastante infrecuente, existiendo cerca de 150 reportes en la literatura. La asociación de ambas entidades se observa en $16 \%$ a $71 \%$ de los casos de hamartoma mamario.

La intención de esta publicación es reportar un caso clínico de hamartoma mamario con PASH.

\section{Caso clínico}

Mujer de 44 años, sin antecedentes médicos relevantes. Acude por 4 años de evolución de mastodinia derecha asociada a tumor palpable expansivo de mama derecha, acompañado de telorrea verdosa durante el último año. A la inspección no se ve tumor, retracciones ni ulceraciones. Al examen se palpa un nódulo bien delimitado móvil de $5,5 \times 5 \mathrm{~cm}$, en axila ipsilateral presenta una adenopatía móvil.

La mamografía evidencia una imagen pseudo nodular de mama derecha asociada a mastopatía fibroquística (MFQ) informada como BIRADS 0 (Figura 1). La ecografía muestra un tumor de 1,9 x 2,3 x 1,8 cm, heterogéneo, irregular, hipoecogénico, con escasa vascularización, informado como BIRADS US: 5, (Figura 2).

Considerando contexto clínico se presume probable patología maligna y se solicita evaluación por comité oncológico, decidiéndose extensión de estudio con biopsia CORE, tomografía de tóraxabdomen-pelvis y cintigrama óseo. El cintigrama muestra hipercaptación de T9, la tomografía muestra nódulo inespecífico en lóbulo superior de pulmón derecho y quistes inespecíficos en ambos campos pulmonares, la biopsia CORE informa PASH, micro- calcificaciones no detectadas y mastopatía fibrosa. Se decide completar estudio con RNM contrastada con gadolinio, la cual no muestra captación patológica en mama derecha ni adenopatías. Informada como BIRADS 3.

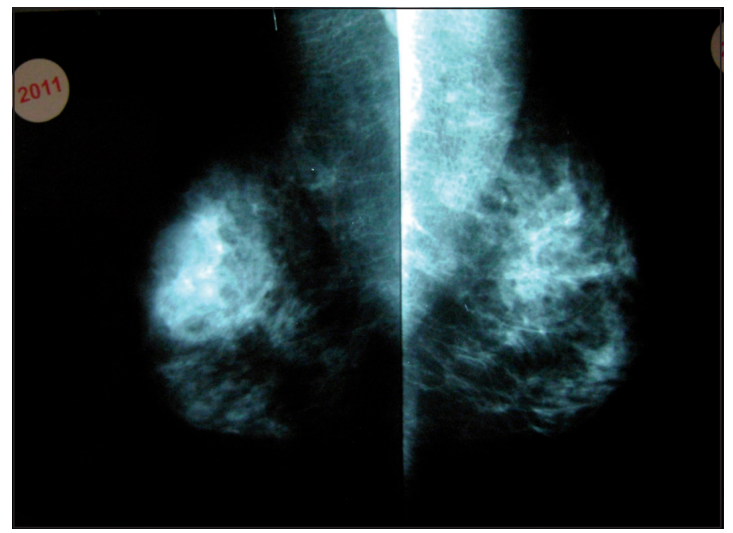

Figura 1. Mamografía preoperatoria de la paciente. Se evidencia imagen radiopaca pseudonodular en CSE de mama derecha asociado a mastopatía fibroquística. Informado como BIRADS 0 .

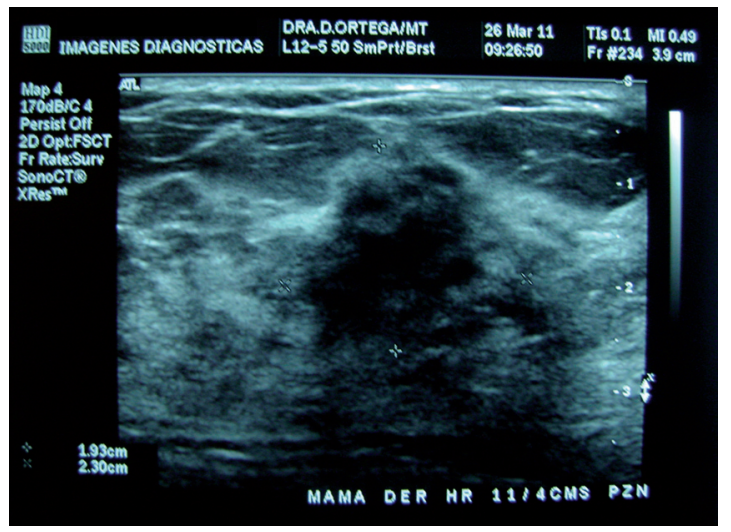

Figura 2. Ecografía preoperatoria de la paciente. Se evidencia una imagen de $1,9 * 2,3 * 1,8 \mathrm{~cm}$ en CSE de mama derecha, de ecogenicidad heterogénea, irregular, con escasa vascularización al doppler. Informado como BIRADS US 5. CSE: Cuadrante supero externo. 


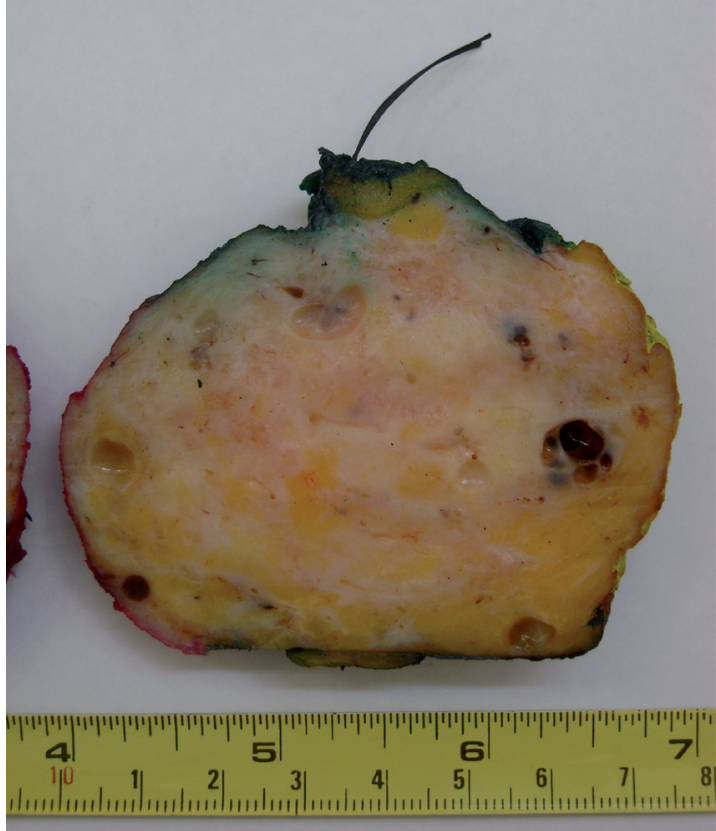

Figura 3. Imagen macroscópica de la pieza quirúrgica resecada. Aprecie la lesión sólida homogénea color blanco-grisáceo al centro de la muestra y las lesiones quísticas circundantes.

Considerando el diagnóstico histológico y la sospecha clínica de cáncer, se realiza mastectomía parcial sin incidentes, en el post operatorio la paciente evoluciona satisfactoriamente siendo dada de alta al $2^{\circ}$ día post operatorio. El informe de la biopsia diferida confirma la presencia de PASH pero en el contexto de hamartoma mamario, en el cual prácticamente todo el estroma perilobulillar presenta características de PASH (Figuras 3 y 4). En los controles posteriores alejados no se objetivan complicaciones ni recidivas.

\section{Discusión}

\section{Hiperplasia estromal pseudoangiomatosa}

La PASH, reportada inicialmente en 1986 por Vuitch, es una entidad clínica benigna, caracterizada por una proliferación mesenquimática del tejido mamario, dando forma a pseudoangiosomas ${ }^{1}$. Si bien es una patología bien definida, su fisiopatología es poco comprendida, la teoría más aceptada en la literatura postula una respuesta alterada de los miofibroblastos al estímulo hormonal, particularmente progestágeno ${ }^{2}$.

La tasa de presentación incidental prospectiva en estudios de mastectomías parciales o totales es
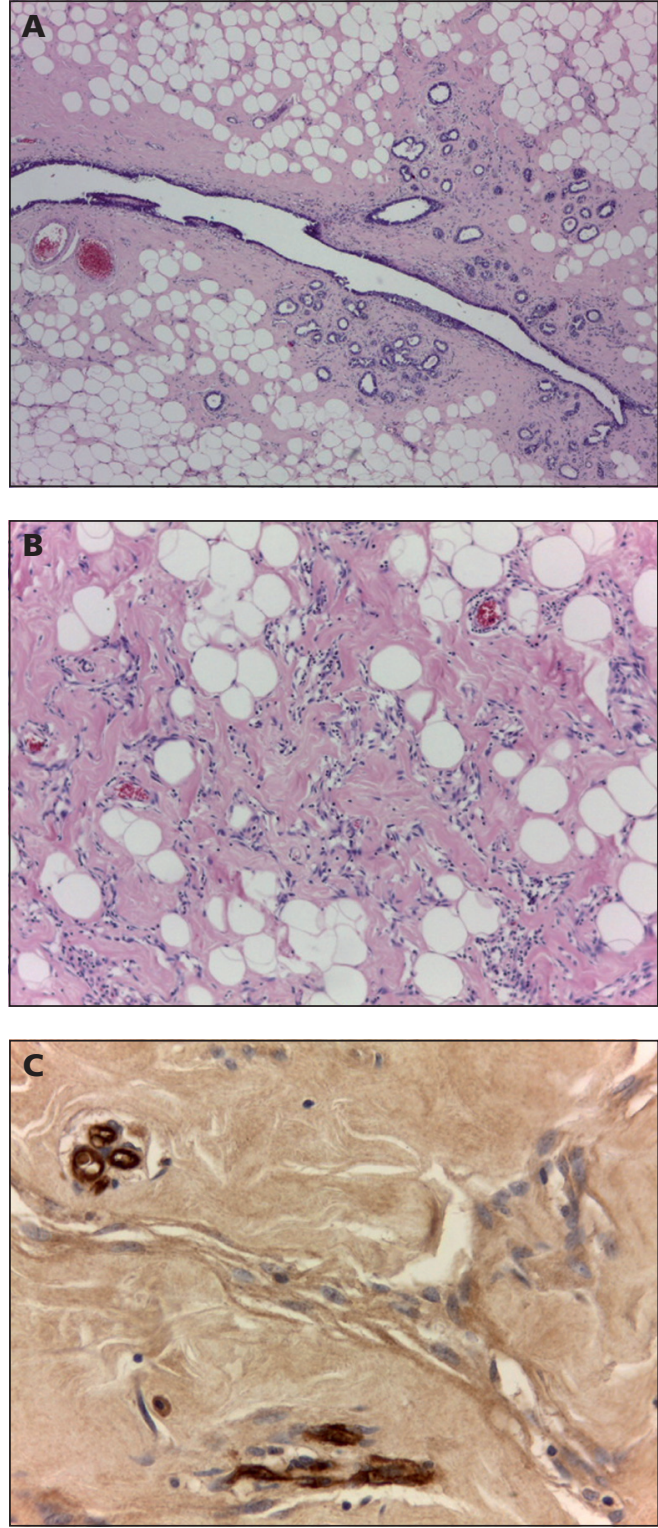

Figura 4. Imagen microscópica de la pieza quirúrgica resecada. Tres cortes de microscopia. A) Hamartoma: Aumento x 40, tinción de HE. Tejido mamario maduro y normal pero dispuesto de manera desordenada, con distribución no sistemática de tejido fibroso y adiposo. Conductos y lobulillos de estructura muy variable, vasos sanguíneos de tamaño mayor a lo esperado. B) PASH: Aumento x 100, tinción de HE. A mayor aumento se aprecia que todo el tejido fibroso presenta múltiples surcos que se anastomosan, sin glóbulos rojos en su interior, en parte flanqueados por células fusadas de aspecto benigno. C) PASH, aumento de pseudoangiosomas: Aumento x 400, tinción de F8. Estos espacios no son vasos sanguíneos, ni las células que lo flanquean son endoteliecitos, estas células son miofibroblastos, por lo tanto, no se tiñen con factor VIII que tiñe los vasos sanguíneos. 
de hasta un $23 \%$, también puede presentarse en hombres, reportándose tasas prospectivas de hasta un $47 \%$ en el estudio de ginecomastia. La presentación clínica es en mujeres entre 30 y 40 años, como hallazgo incidental en estudios anatomopatológicos de otros cuadros clínicos. La presentación nodular es muy infrecuente, su incidencia no ha sido descrita, tiende a manifestarse en forma unilateral, de crecimiento rápido, bien delimitado, de consistencia firme y móvil ${ }^{2-4}$.

Mamográficamente suele apreciarse una imagen radiopaca, bien delimitada, ovalada sin calcificaciones. Ecográficamente los hallazgos son imágenes sólidas, hipoecogénicas, homogéneas y bien delimitadas. La resonancia magnética muestra lesiones isointensas en T1 e imágenes lineares reticulares en T2. La tomografía computada no se describe como una herramienta útil. La literatura concuerda en que los hallazgos imagenológicos son variados y requieren del estudio histológico confirmatorio ${ }^{5,6}$.

La obtención de la muestra se realiza bajo biopsia excisional o por biopsia CORE guiada ecográficamente, presentando esta última una sensibilidad de un $83 \%{ }^{5}$.

El estudio anatomopatológico macroscópico evidencia tumores de hasta $20 \mathrm{~cm}$ de diámetro, de aspecto ovalado, bien circunscrito, no encapsulado, y de superficie lisa, en su interior destaca un color blanquecino grisáceo homogéneo con focos quísticos. El estudio microscópico revela proliferación estromal fibrosa con formas de espacios irregulares "pseudovasculares" anastomosados sin contenido en su interior, recubiertos por células planas discontinuas en formación "pseudoendotelial", no se evidencian hallazgos de atipía, esta proliferación fibrosa corresponde a células fibromioelásticas del estroma perilobulillar. Los marcadores inmunohistoquímicos presentes son CD34, vimentina, actina de músculo liso (SMA), BCL-2; siendo negativos para CD31, citoqueratina, S100 y antígeno de factor von Willebrand ${ }^{2}$.

El diagnóstico diferencial de esta patología incluye: fibroadenoma, hemangiosarcoma de bajo grado, miofibroblastoma y hamartoma ${ }^{2}$.

\section{Hamartoma mamario}

El hamartoma mamario fue reportado en 1971 por Arrigoni. Es una patología benigna, en los últimos años ha aumentado su diagnóstico.

$\mathrm{Su}$ incidencia es de 0,1 a $0,7 \%$, aunque se estima mayor. La presentación puede darse entre los 15 y los 88 años. La clínica es de un tumor sólido, blando, bien delimitado, móvil, no adherido, indoloro ${ }^{7}$.

Mamográficamente los hallazgos son relativamente constantes, con imágenes radiopacas con áreas radiolúcidas, ovaladas, bien definidas y encap- suladas. Ecográficamente se puede apreciar bordes bien definidos con ecorefringencia heterogénea y desplazamiento de parénquima adyacente. La RNM objetiva intensidades grasas con un halo hipointenso y captación heterogénea del contraste.

La toma de muestra para biopsia se realiza a través de punción de aspiración con aguja fina y biopsia CORE, sin embargo, el rendimiento de éstas es variable y puede verse dificultado aun más si no se cuenta con los antecedentes clínicos y radiológicos ${ }^{8}$.

No existen criterios histológicos diagnósticos específicos. Sin embargo, la presencia de lóbulos con estroma fibrograso, estroma laxo interlobar y elementos epiteliales (todo ello con una relación no sistemática) es común ${ }^{7}$.

El tratamiento definitivo de ambas patologías es la resección quirúrgica con márgenes negativos sin necesidad de otras terapias asociadas. Existe un reporte aislado de manejo de PASH nodular bajo esquema de Tamoxifeno ${ }^{9}$. La recurrencia tumoral ipsi o contralateral depende de la serie analizada, variando desde $0 \%$ hasta $22 \%{ }^{2}$.

En el caso clínico se expone inicialmente una PASH nodular que finalmente concluyó en la asociación de hamartoma con PASH, la cual se describe en un $16 \%$ a un $71 \%$ de los casos 7 . Si bien ambas entidades son benignas y no se ha demostrado malignización hasta la fecha, es importante destacar su forma de presentación como probable patología maligna determinando así un diagnóstico diferencial del cáncer de mama. Aunque consista en una entidad infrecuente es necesario conocer el comportamiento de esta entidad para ofrecer las mejores alternativas diagnóstico-terapéuticas.

Ante el diagnóstico de PASH en la biopsia CORE, y siendo esto tan frecuente como hallazgo incidental en múltiples otras mastopatías, tanto malignas como benignas, es muy importante la correlación con la imagenología para determinar que la lesión investigada en cada caso fue realmente muestreada en la CORE.

En la literatura no existe una recomendación universal para el seguimiento de estos pacientes, sin embargo, considerando el riesgo de recurrencia y la costoefectividad del estudio imageneológico, parece lógico recomendar controles clínicos, mamográficos y ecográficos, aparentemente la RNM no presentaría un beneficio sustantivo, sin embargo, no existen ensayos clínicos prospectivos que respalden esta postura.

\section{Referencias}

1. Vuitch MF, Rosen PP, Erlandson RA. Pseudoangiomatous hyperplasia of mammary stroma. Hum Pathol. 1986;17:185-91. 
2. Virk RK, Khan A. Pseudoangiomatous stromal hyperplasia: an overview. Arch Pathol Lab Med. 2010; 134:1070-4.

3. Ibrahim RE, Sciotto CG, Weidner N. Pseudoangiomatous hyperplasia of mammary stroma. Some observations regarding its clinicopathologic spectrum. Cancer 1989;63:1154-60.

4. Milanezi MFG, Saggioro FP, Zanati SG, Bazan R, Schmitt FC. Pseudoangiomatous hyperplasia of mammary stroma associated with gynecomastia. J Clin Pathol. 1998;51:204-6.

5. Wieman SM, Landercasper J, Johnson JM. Tumoral pseudoangioma- tous stromal hyperplasia of the breast. Am Surg. 2008;74:1211-4.

6. Yoo K, Woo OH, Yong HS. Fast-growing pseudoan- giomatous stromal hyperplasia of the breast: report of a case. Surg Today 2007;37:967-70.

7. Tse GM, Law BK, Ma TK, Chan AB, Pang LM, Chu WC, et al. Hamartoma of the breast: a clinicopathological review. J Clin Pathol. 2002;55:951-4.

8. Ruiz-Tovar J, Reguero-Callejas ME, Arano-Bermejo JI, González-Palacios F, Cabañas-Navarro L. Hamartoma mamario. Cir Esp. 2006;79:186-8.

9. Sandhya Pruthi. Tamoxifen in the Management of Pseudoangiomatous Stromal Hyperplasia. The Breast Journal 2001;7:434-9.

10. Powell CM, Cranor ML, Rosen PP. Pseudoangiomatous stromal hyperplasia (PASH): a mammary stromal tumor with myofibroblastic differentiation. Am J Surg Pathol. 1995;19:270-7. 